\title{
A Nova Gestão Pública no Contexto da Formação Continuada de Professores: o Caso do Mestrado Nacional Profissional em Ensino de Física
}

\section{The New Public Management Policy in the Context of Continuing Teacher Education: the Case of the National Professional Master's Degree in Physics Teaching}

Paulo Vinícius Rebeque ${ }^{1}$

Fernanda Ostermann ${ }^{2}$

Sofia Viseu ${ }^{3}$

\author{
'Instituto Federal de Educação' Ciência e Tecnologia do Rio Grande do Sul (IFRS), Bento Gonçalves, RS, Brasil. \\ Autor correspondente: paulo.rebeque@bento.ifrs.edu.br \\ ${ }^{2}$ Universidade Federal do Rio Grande do Sul (UFRS), Instituto de Física, Porto Alegre, RS, Brasil. \\ ${ }^{3}$ Universidade de Lisboa (ULisboa), Instituto de Educação, Lisboa, Portugal.
}

Resumo: Neste artigo analisamos os processos de criação e expansão dos Mestrados Profissionais em Ensino, cursos stricto sensu de formação continuada de professores, no contexto da Nova Gestão Pública. Em especial, tecemos investigações sobre o Mestrado Nacional Profissional em Ensino de Física criado em 2013 sob coordenação da Sociedade Brasileira de Física. Com isso, colocamos em evidência a organização administrativa e as práticas pedagógicas pré-determinadas do Mestrado Profissional e seu alinhamento aos princípios da Nova Gestão Pública, isto é, da adoção de uma lógica empresarial para a formação continuada de professores. Por fim, com base nos números referentes ao êxito acadêmico das três primeiras turmas, tecemos reflexões, por um lado, sobre o alcance da formação em larga escala que este programa tem atingido e, por outro lado, sobre possíveis caminhos de abertura administrativa não necessariamente alinhada à nova gestão pública.

Palavras-chave: Mestrado profissional; Ensino de física; Curso de pós-graduação; Gestão pública.

Abstract: In this article, we analyze the processes of creation and expansion of the Professional Master's Degrees in Teaching, stricto sensu continuing teacher education programs, in the context of New Public Management. In particular, we did research on the National Master's Degree in Physics Teaching created in 2013 under the coordination of the Brazilian Society of Physics. Thus, we highlight the administrative organization and the standardized pedagogical practices of rofessional Master's Degree and its alignment with the principles of New Public Management, that is, of adopting a business logic for continuing teacher education. Finally, based on the numbers concerning the academic success of the first three groups that completed the Professional Master's Degree, we reflect on the scope of the large-scale training that this program has been able to promote and, on the other hand, on possible paths of administrative transformation that are not in line with the new public management.

Keywords: Professional master's degree; Physics teaching; Postgraduate course; Public management.

Recebido em: $31 / 10 / 2018$

Aprovado em: 07/02/2020 


\section{Introdução}

A partir do ano de 2010, com os cursos de Mestrado Profissional em Ensino (MPE) (MOREIRA, 2004) - sobretudo na subárea de Ensino de Ciências e Matemática (ECM) (MOREIRA; NARDI, 2009) - consolidados como prática acadêmica na pós-graduação brasileira (REBEQUE; OSTERMANN; VISEU, 2017; VILLANI et al., 2017), a Coordenação de Aperfeiçoamento de Pessoal de Nível Superior (CAPES) deu início à política de implementação dos Programas de Mestrado Profissional em Rede Nacional.

Tratam-se de programas de pós-graduação stricto sensu que objetivam a formação continuada de professores em larga escala de específicas áreas do conhecimento, tais como Matemática, Física, História, Artes, entre outras ${ }^{1}$. No contexto normativo desses programas, os estudantes precisam estar em pleno exercício de docência na Educação Básica, por isso, os entendemos como discentes/professores.

Em termos de organização estrutural, os programas em rede nacional são formados por um conjunto de cursos, denominados polos regionais (PR), que seguem normas específicas, estabelecidas por um Conselho Nacional próprio, nomeado pela entidade ou Instituição de Ensino Superior (IES) que coordena o programa, sendo financiado em partes com recursos da CAPES, principalmente com bolsas de estudos para os discentes/professores.

Dentre os programas em rede nacional existentes está o Mestrado Nacional Profissional em Ensino de Física (MNPEF), formado por 60 Polos Regionais espalhados por todo o país e que teve início em Agosto de 2013 sob coordenação da Sociedade Brasileira de Física (SBF). Essencialmente, o MNPEF objetiva a qualificação em larga escala de professores atuantes na disciplina de Física, em especial, a partir do desenvolvimento de produtos e técnicas que envolvam, necessariamente, o ensino de Física na Educação Básica (MOREIRA; STUDART; VIANNA, 2016; NASCIMENTO, 2013).

Naturalmente que o Mestrado Nacional Profissional em Ensino de Física, assim como os cursos de Mestrado Profissional em Ensino e, posteriormente, os programas em rede nacional, não surgiu de maneira repentina no cenário da pós-graduação brasileira. Estes programas, antes de tudo, fazem parte do contexto de reformas e transformações educacionais que o Brasil tem adotado nos últimos anos, frutos de práticas alinhadas à Nova Gestão Pública (NGP) e ao modelo de globalização neoliberal (ANDERSON, 2017; CÓSSIO, 2018; OLIVEIRA, 2015). Neste cenário da NGP, a formação continuada de professores tem sido balizada pela concepção da profissionalização docente, isto é, em um modelo standard de formação, acelerado e em larga escala, que prioriza a transmissão de conhecimentos já produzidos, manifestado, sobretudo, na padronização do currículo (SANTOS; DINIZ-PEREIRA, 2016).

Neste artigo, ao tomarmos o Mestrado Nacional Profissional em Ensino de Física (MNPEF) como objeto de investigação, resgatamos as características deste programa, Regimento Geral (SOCIEDADE BRASILEIRA DE FÍSICA, 2015) e Matriz Curricular Nacional (SOCIEDADE BRASILEIRA DE FÍSICA, 2014), assim como de seu contexto de criação (REBEQUE; OSTERMANN; VISEU, 2018), de modo a tecermos reflexões com bases nas ações e práticas desenvolvidas nos cinco primeiros anos do MNPEF, principalmente, sob a ótica da Nova Gestão Pública e do modelo de formação docente intrínseco deste programa. 
Em primeiro lugar, destacamos os pressupostos da Nova Gestão Pública no contexto da formação continuada de professores manifestados nos cursos de Mestrado Profissional em Ensino e nos programas em rede nacional. Com isso, pretendemos discutir a introdução de uma lógica empresarial, decorrente da adoção dos princípios da Nova Gestão Pública na ação de um organismo da administração pública, a Coordenação de Aperfeiçoamento de Pessoal de Nível Superior (CAPES), instância governamental que regula, e também financia, a pós-graduação no Brasil.

$\mathrm{Na}$ sequência, apresentamos dados quantitativos referentes ao êxito acadêmico dos discentes/professores do Mestrado Nacional Profissional em Ensino de Física para comentarmos o alcance da formação em larga escala deste programa. Em outras palavras, apresentamos, em números, as vagas ofertadas nos editais para a seleção de ingresso e os respectivos concluintes, discentes/professores que cumpriram todas as exigências do programa e, naturalmente, foram titulados mestres em Ensino de Física.

Para encerrar, colocamos no debate as práticas pedagógicas pré-determinadas deste programa no contexto da formação continuada de professores pautada na Nova Gestão Pública. Em especial, com base em investigações realizadas sobre o próprio Mestrado Nacional Profissional em Ensino de Física e considerando os cinco primeiros anos de sua existência, buscamos ampliar nossas reflexões no caminho de uma administração aberta e plural e, consequentemente, de possibilidades de maior autonomia para os polos regionais (PR), viabilizando, assim, dinâmicas formativas não padronizadas, essenciais para a formação continuada de professores (IMBERNÓN, 2010).

\section{A Nova Gestão Pública e os Mestrados Profissionais em Ensino}

No contexto brasileiro, desde o final da década de 1980 e início dos anos 1990 as reformas educacionais têm sido justificadas a partir das mudanças econômicas impostas pela globalização (MAUÉS, 2003). Com isso, tivemos grandes impactos na formação e no trabalho docente, que passou a ser fortemente estruturado no desenvolvimento de competências sob o enfoque de um currículo instrumental, além da relação entre o desempenho do professor e de seus alunos, podendo esta relação ser avaliada, mensurada e certificada (DIAS; LOPES, 2003; FREITAS, 2007). É justamente neste cenário que se configura a Nova Gestão Pública na educação pública: da importação de práticas derivadas do setor privado, da busca por modernização e eficiência, tal como o modelo empresarial (CÓSSIO, 2018; OLIVEIRA, 2015).

Vale lembrarmos que a Nova Gestão Pública (GNP) não é uma entidade homogênea, mas resulta da sedimentação progressiva de novas maneiras de pensar a organização administrativa (OLIVEIRA, 2015). Nas palavras de Cóssio, (2018, p. 68), a Nova Gestão Pública

[...] busca inserir na raiz do sistema público conceitos e práticas voltados a eficiência, eficácia, competitividade, administração por objetivos, meritocracia e demais concepções oriundas de um meio em que a finalidade é a obtenção de lucro e que, portanto, dispensa pouca atenção para as finalidades sociais.

Robertson (2013), por exemplo, evidencia que na educação pública, quando adotada a lógica empresarial, as ações do governo não são, necessariamente, realizadas por ele próprio, mas sim por forte influência de agentes não governamentais. Ou seja, trata-se de uma nova forma de privatização: do deslocamento da concepção de "educação superior" para "educação terciária" (da tríade ensino, pesquisa e extensão, para a vertente exclusiva 
do ensino, de transmissão de conteúdos), de reconfiguração do modelo de gestão nas instituições educacionais públicas, agora, embasado no modelo empresarial de gestão. (LIMA, 2011).

Nesse contexto, podemos destacar as principais implicações na formação e no trabalho docente, quais sejam: currículos e práticas pedagógicas homogeneizadas, aligeiramento dos processos, intensificação do trabalho docente e ênfase na gestão por resultados. Como exemplo, temos os estudos de Evangelista e Shiroma (2007) sobre o papel das agências multilaterais nas políticas de formação docente na América Latina e Caribe, e de Casagrande, Pereira e Sagrillo (2014), sobre o Banco Mundial e as políticas de formação de professores no Brasil.

No caso específico da formação continuada de professores via cursos de Mestrado Profissional em Ensino, que surgiram no Sistema Nacional de Pós-Graduação (SNPG) em 2002, percebemos a forte ênfase destes cursos nos conteúdos disciplinares voltados para o ensino, não na pesquisa (MOREIRA, 2004), adotando, assim, currículos e práticas pedagógicas homogeneizadas em todo o país. Ainda, por serem cursos distintos dos já existentes cursos de mestrado acadêmico (MA), ao menos na subárea de Ensino de Ciências e Matemática, os cursos de Mestrado Profissional em Ensino devem estar de acordo com as características descritas no quadro 1, segundo Moreira e Nardi (2009).

\section{Quadro 1 - Principais características dos cursos de Mestrado Profissional em Ensino (MPE) na subárea Ensino de Ciências e Matemática (ECM)}

\begin{tabular}{|c|c|}
\hline 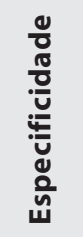 & $\begin{array}{l}\text { Os cursos de Mestrado Profissional em Ensino pertencentes a subárea de ECM podem atender em um único } \\
\text { curso um feixe de disciplinas, como por exemplo, Ensino de Ciências e Matemática (Ensino de Ciências da } \\
\text { Natureza, Ensino Científico e Tecnológico), ou duas disciplinas, como é o caso de cursos como Ensino de Física } \\
\text { e Química, Ensino de Física e Matemática, ou ainda, estar centrado em uma especíica disciplina: Ensino de } \\
\text { Biologia, Ensino de Física, Ensino de Matemática, etc. }\end{array}$ \\
\hline 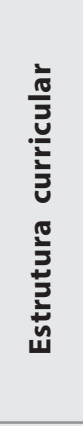 & $\begin{array}{l}\text { - De } 30 \text { a } 50 \text { \% da carga horária total dos cursos devem ser em disciplinas obrigatórias de conteúdos, conforme } \\
\text { especificidade do curso. Além do mais, as disciplinas devem ter ementas próprias, bibliografias atualizadas } \\
\text { e direcionadas ao ensino e a transposição didática. } \\
\text { - A matriz curricular precisa contemplar disciplinas de natureza pedagógica e epistemológica. Estas disci- } \\
\text { plinas, além de estarem integradas com as disciplinas de conteúdo, devem focar em aspectos do ensino e } \\
\text { da aprendizagem, de modo a subsidiarem a elaboração de novas abordagens e estratégias inovadoras de } \\
\text { ensino a serem implementadas nas salas de aulas. } \\
\text { - Há também de se destinar um espaço para o acompanhamento da prática profissional do estudante do } \\
\text { MPECM, o chamado estágio supervisionado. Trata-se de um momento oportuno para que o orientador } \\
\text { acompanhe o mestrando em seu exercício docente. }\end{array}$ \\
\hline 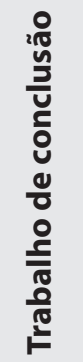 & $\begin{array}{l}\text { A natureza do trabalho de conclusão do Mestrado Profissional em Ensino (MPE) é distinta da do Mestrado } \\
\text { Acadêmico (MA). No caso do MPE, o trabalho de conclusão é formado por uma dissertação e por um produto } \\
\text { educacional. Em resumo, o mestrando deve desenvolver um processo ou produto de natureza educacional e } \\
\text { implementá-lo em condições reais de ensino (sala de aula, espaços informais, cursos de curta duração, etc.) e } \\
\text { relatar toda essa experiência (elaboração, implementação e avaliação) no texto da dissertação. Neste contexto, } \\
\text { o produto educacional é considerado uma produção técnica e deve ser disponibilizado em diversos meios de } \\
\text { comunicação para que outros possam ter acesso. }\end{array}$ \\
\hline 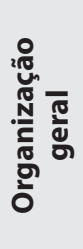 & $\begin{array}{l}\text { - Como cursos de pós-graduação destinado aos professores que estão em pleno exercício de suas atividades } \\
\text { docentes, os MPECM devem, necessariamente, concentrar suas atividades didáticas em um ou dois dias da } \\
\text { semana, ou ainda em cursos condensados nos períodos de férias escolares. } \\
\text { - A titulação ocorrerá após o cumprimento das disciplinas da matriz curricular e da defesa e aprovação do } \\
\text { trabalho de conclusão de curso, de modo que o tempo para a realização de todas essas exigências deve } \\
\text { ocorrer entre } 24 \text { meses no mínimo e } 36 \text { meses no máximo. }\end{array}$ \\
\hline
\end{tabular}


De fato, podemos perceber uma manifestação da Núcleo de Pós-graduação (NPG) no modelo homogeneizado de formação continuada de professores proposto pelos cursos de Mestrado Profissional de Ensino (MPE), em especial, por considerarem fortemente aspectos disciplinares, uniformes e supostamente "objetivos", o chamado modelo de treinamento (IMBERNÓN, 2010). Ou ainda, o modelo de "preenchimento de lacunas", pois parte do pressuposto de que "[...] a formação inicial não foi suficiente para que o professor desenvolva plenamente a tarefa docente" (SAUERWEIN; DELIZOICOV, 2008, p. 457). Ademais, soma-se o viés tecnicista e instrumental do trabalho de conclusão de curso para o Mestrado Profissional em Ensino que, na subárea de Ensino de Ciências e Matemática (ECM), caminha na direção da simples aplicação de um produto educacional seguindo concepções de senso comum no ambiente restrito da sala de aula (OSTERMANN; REZENDE, 2009).

Não obstante, a lógica empresarial como prática na formação continuada de professores também se justifica pelo potencial de oferta em larga escala. Por isso, desde o surgimento dos cursos de Mestrado Profissional em Ensino, observamos intenso crescimento dessa modalidade de mestrado (REBEQUE; OSTERMANN; VISEU, 2017), sobretudo no âmbito dos programas em rede nacional, que podem vir a ofertarem entre 12 e 13 mil vagas anuais em seu conjunto (CAPES, 2017).

Nesse cenário da Nova Gestão Pública, de importação de práticas da administração privada na gestão educacional (OLIVEIRA, 2015), percebemos o quanto restrito está o debate acerca da formação continuada de professores via cursos de Mestrado Profissional em Ensino, pensada, quase que exclusivamente, nos conteúdos disciplinares abordados no ambiente fechado da sala de aula. Em um primeiro nível, temos notória desarticulação dos Mestrado Profissional em Ensino com os cursos de formação inicial, isto é, uma formação centrada na "atualização" de conteúdos disciplinas e que deixa de lado a formação pedagógica, política e social dos professores. Em um segundo nível, pelo caráter inicial de terminalidade atribuído ao Mestrado Profissional em Ensino (MOREIRA, 2004), posteriormente suprimido com a criação do doutorado profissional (DP) (BRASIL, 2017, 2019) ${ }^{2}$, pensado sem um devida contextualização com políticas públicas amplas e contínuas que envolvam o todo da carreira dos professores.

Em outras palavras, devemos ter um olhar completo e colocar todas as questões que envolvem a atividade docente no debate, não apenas a oferta em larga escala. Conforme nos atesta Kuenzer (2011, p. 672): "[...] a política de formação [de professores] só tem sentido quando integrada à estruturação da carreira docente, à política salarial que assegure a dignidade do professor e à garantia de condições adequadas de trabalho". Neste quadro da formação continuada de professores identificamos um estreito alinhamento entre as práticas adotadas nos cursos de Mestrado Profissional em Ensino em rede nacional com os princípios da Nova Gestão Pública, o que nos distancia do ideário de uma política pública educacional "[...] que contemple de forma articulada e prioritária a formação inicial, continuada e condições de trabalho, salário e carreira" (FREITAS, 2007, p. 1204).

${ }^{2}$ Em consulta ao Sistema Nacional de Pós-Graduação (SNPG) constatamos que há 49 cursos de doutorado profissional, dos quais oito são na área de Ensino: sete na subárea de Ensino de Ciências e Matemática e um na subárea de Ensino em Saúde. Disponível em: https://tinyurl.com/y8z4a32j. Acesso em: 20 maio 2020. 


\section{Sobre as principais características do Mestrado Nacional Profissional em Ensino de Física (MNPEF)}

No tocante aos poloa regionais (PR) que compõem o Mestrado Nacional Profissional em Ensino de Física, o processo de criação foi regulado pela Sociedade Brasileira de Física por meio de editais para este propósito. Todo esse processo foi conduzido pela Comissão de PósGraduação (CPG) do MNPEF, sendo que, somente nos três primeiros anos, foram criados 60 polos regionais em todo o território nacional (mais informações no anexo A). Todos possuem regimento próprio em conformidade com o Regimento Geral do MNPEF (SOCIEDADE BRASILEIRA DE FÍSICA, 2015) e Matriz Curricular Nacional (SOCIEDADE BRASILEIRA DE FÍSICA, 2014), assim como seguem orientações para o trabalho de conclusão, composto por uma dissertação e um produto educacional (MOREIRA, 2015; MOREIRA; STUDART, 2015).

A partir da acelerada expansão de 60 Polos Regionais hospedados em Instituições de Ensino Superior Públicas, temos o Amapá como único estado brasileiro que não oferta o Mestrado Nacional Profissional em Ensino de Física. Em termos de região, a Nordeste figura com o maior quantitativo, com 21 polos regionais, seguido pelas regiões Sudeste (15), Sul (10), Norte (9) e Centro-Oeste (5). Nessa perspectiva, podemos dizer que o Mestrado Nacional Profissional em Ensino de Física tem cumprido com o seu papel de oferecer cursos de formação continuada para professores de Física atuantes na Educação Básica. Entretanto, acreditamos ser oportuna a reflexão sobre a padronização dos cursos vinculados ao programa, isto é, de polos regionais espalhados por todo território nacional estarem atrelados às práticas acadêmicas pré-determinadas: currículo nacional e trabalho de conclusão.

Importa-nos lembrar que na época de criação do Mestrado Nacional Profissional em Ensino de Física, três cursos de Mestrado Profissional em Ensino de Física (MPEF) já existiam no país, quais sejam: Universidade Federal do Rio Grande do Sul (UFRGS), criado em Março de 2002 - sendo assim o primeiro curso de MPEF do Brasil -, Universidade Federal do Rio de Janeiro (UFRJ), reconhecido pelo Conselho Nacional de Educação (CNE) em Dezembro de 2007, e Universidade Federal do Espírito Santos (UFES), com início das atividades acadêmicas em Agosto de $2011^{3}$. Com isso, o Mestrado Nacional Profissional em Ensino de Física buscou se espelhar nas experiências destes cursos, principalmente nos 'costumes' para o trabalho de conclusão e nas disciplinas da matriz curricular do curso pioneiro da UFRGS.

Em nossa percepção, tal organização do Mestrado Nacional Profissional em Ensino de Física está alinhada aos pressupostos da Nova Gestão Pública (CÓSSIO, 2018), pois, mesmo com todos os Polos Regionais hospedados em Instituição de Ensino Superior Pública, a homogeneização das práticas acadêmicas deste programa se mostra evidente. Em outras palavras, ainda que seja administrado pela Sociedade Brasileira de Física (constituída como uma associação sem fins lucrativos) e financiado pela CAPES (agência governamental), há no MNPEF uma nítida importação da lógica empresarial, de busca por resultados eficientes, para o contexto da formação continuada de professores.

Em especial, na organização da matriz curricular nacional do Mestrado Nacional Profissional em Ensino de Física (SOCIEDADE BRASILEIRA DE FÍSICA, 2014), conforme apresentado no quadro $\mathbf{2}$, damos destaque às disciplinas que se concentram nos conteúdos

${ }^{3}$ Dos três cursos citados, dois estão integrados ao programa em rede nacional da Física: o curso da Universidade Federal do Rio de Janeiro (UFRJ) é o polo regional 22 (agregado em 2014) e o curso da Universidade Federal do Espírito Santo (UFES) é o polo regional 12, presente desde 2013. Por fim, o curso da Universidade Federal do Rio Grande do Sul (UFRGS) deixou de ofertar vagas no ano de 2016. 
de Física e ocupam $50 \%$ da carga horária total do programa, justamente dentro da lógica do modelo de 'preenchimento de lacunas'.

Quadro 2 - Matriz curricular nacional do Mestrado Nacional Profissional em Ensino de Física

\begin{tabular}{|c|c|c|c|}
\hline \multicolumn{3}{|r|}{ Nome da disciplina } & Carga horária \\
\hline \multirow{7}{*}{ 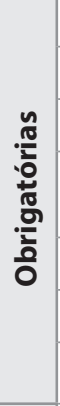 } & \multicolumn{2}{|c|}{ Termodinâmica e Mecânica Estatística } & 60 \\
\hline & \multicolumn{2}{|c|}{ Eletromagnetismo } & 60 \\
\hline & \multicolumn{2}{|c|}{ Mecânica Quântica } & 60 \\
\hline & \multicolumn{2}{|c|}{$\begin{array}{l}\text { Física Contemporânea (Física de Partículas, Espaço-Tempo, Física da Matéria Condensada, Biofísica, } \\
\text { dependendo do Polo Regional) }\end{array}$} & 60 \\
\hline & \multicolumn{2}{|c|}{ Fundamentos Teóricos em Ensino e Aprendizagem } & 30 \\
\hline & \multicolumn{2}{|c|}{ Marcos no desenvolvimento da Física } & 30 \\
\hline & \multicolumn{2}{|c|}{ Estágio Supervisionado } & 60 \\
\hline \multirow{4}{*}{ 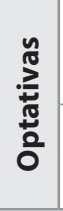 } & \multirow{2}{*}{$\begin{array}{l}\text { Experimental / } \\
\text { Computacional }\end{array}$} & Atividades experimentais para o Ensino Médio e Fundamental & 60 \\
\hline & & Atividades computacionais para o Ensino Médio e Fundamental & 60 \\
\hline & \multirow{2}{*}{ Ensino } & Processos e sequências de Ensino e Aprendizagem em Física no Ensino Médio & 60 \\
\hline & & Física no Ensino Fundamental em uma perspectiva multidisciplinar & 60 \\
\hline
\end{tabular}

Fonte: Sociedade Brasileira de Física (2014).

De modo geral, as características do currículo dos cursos de Mestrado Profissional em Ensino na subárea de Ensino de Ciências e Matemática, na interpretação de Villani (2016), não podem ser entendidas como o resultado de escolhas racionais e neutras dos agentes públicos, mas sim como o resultado de lutas políticas no campo acadêmico entre três grupos de pesquisadores: em Ciências, em Educação e em Ensino de Ciências e Matemática. Para este autor, cada grupo acima mencionado, seguindo seus pressupostos, disputam a influência e o controle das iniciativas no meio acadêmico.

No caso do Mestrado Nacional Profissional em Ensino de Física, sendo a Sociedade Brasileira de Física (SBF) formada por maioria de pesquisadores em Ciências, que por sua vez defendem uma perspectiva disciplinar, sustentando o domínio do conhecimento científico como principal questão para a formação continuada (VILLANI, 2016), prevaleceu a concepção de treinamento, de preenchimento de lacunas, como prática formativa deste programa. Ou seja, o projeto do MNPEF está construído de acordo com as necessidades e interesses de um grupo acadêmico (disputas políticas internas pela hegemonia nas tomadas de decisões), que, convém enfatizarmos, não promoveu um debate amplo e democrático com os outros setores acadêmicos, sobretudo pesquisadores da subárea Ensino de Ciências e Matemática, nos processos de criação e administração deste programa (REBEQUE; OSTERMANN; VISEU, 2018).

Esse fato pode ilustrar as contradições que se dão no interior do próprio governo (do poder executivo), entre grupos que disputam a hegemonia das ações, que, mesmo sem notável intenção, seguem dinâmicas alinhadas à Nova Gestão Pública (OLIVEIRA, 2015), tais como imediatismo nos processos de criação e expansão de Polo Regional e de oferta de vagas, padronização do currículo e das práticas pedagógicas, urgência por indicadores de desempenho, entre outros. Além disso, outra contradição que se apresenta perceptível para nós é o academicismo do Mestrado Nacional Profissional em Ensino de Física, resultando em uma subvalorização da formação continuada, pois pauta-se em uma formação técnica e instrumental que não está, necessariamente, vinculada à prática cotidiana dos professores de Física da Educação Básica (ANTUNES JR.; OSTERMANN; CAVALCANTI, 2019). 


\section{Sobre a oferta de vagas e a formação em larga escala do Mestrado Nacional Profissional em Ensino de Física}

O processo seletivo para o ingresso de discentes/professores no Mestrado Nacional Profissional em Ensino de Física é regido por editais publicados pela Sociedade Brasileira de Física em nível nacional e, na sequência, por editais complementares, onde cada Polo Regional publica as informações de seu respectivo domínio.

A primeira etapa do processo seletivo, de caráter eliminatório, consiste em uma prova escrita, centrada na resolução de problemas de Física, elaborada pela Comissão de Pós-Graduação Nacional e aplicada nacionalmente no mesmo dia e horário. A segunda etapa, de natureza classificatória, consiste em uma defesa de memorial, onde os candidatos apresentam um plano de trabalho para o curso de mestrado e uma descrição da trajetória profissional no respectivo polo regional em que se candidataram.

Dos nove editais de processos seletivos publicados até o final de 2018, temos no quadro 3 os seguintes números de Polos Regionais participantes e de vagas ofertadas.

Quadro 3 - informações referentes aos editais de seleção para o ingresso no Mestrado Nacional Profissional em Ensino de Física

\begin{tabular}{|l|c|c|l|}
\hline Edital & $\begin{array}{c}\text { N. de polos regionais } \\
\text { participantes }\end{array}$ & $\begin{array}{c}\text { N. de vagas } \\
\text { ofertadas }\end{array}$ & \multicolumn{1}{|c|}{$\begin{array}{c}\text { Início previsto para as } \\
\text { aulas }\end{array}$} \\
\hline MNPEF-SBF n. 01/2013 & 21 & 365 & $2^{\circ}$ semestre de 2013 \\
\hline MNPEF-SBF n. 01/2014 & 38 & 483 & $2^{\circ}$ semestre de 2014 \\
\hline MNPEF-SBF n. 01/2015 & 06 & 65 & $1^{\circ}$ semestre de 2015 \\
\hline MNPEF-SBF n. 03/2015 & 54 & 694 & $1^{\circ}$ semestre de 2016 \\
\hline MNPEF-SBF n. 01/2016 (vagas remanescentes) & 12 & 65 & $2^{\circ}$ semestre de 2016 \\
\hline MNPEF-SBF n. 02/2016 & 54 & 666 & $1^{\circ}$ semestre de 2017 \\
\hline MNPEF-SBF n. 01/2017 (vagas remanescentes) & 10 & 53 & $1^{\circ}$ ou $2^{\circ}$ semestre de 2017 \\
\hline MNPEF-SBF n. 02/2017 & 56 & 694 & $1^{\circ}$ semestre de 2018 \\
\hline MNPEF-SBF n. 01/2018 & 54 & 619 & $1^{\circ}$ semestre de 2018 \\
\hline
\end{tabular}

Fonte: elaborado pelos autores.

Em números totais, o Mestrado Nacional Profissional em Ensino de Física já ofertou 3.704 vagas nos processos seletivos, demonstrando novamente que está cumprindo com o seu principal objetivo de proporcionar formação continuada para professores de Física em larga escala. No entanto, os processos seletivos realizados, ao estabelecerem uma prova escrita nacional de caráter eliminatório, promoveram, em nosso entender, uma exclusão de professores (tendo em vista que muitas das vagas ofertadas se tornaram vagas ociosas).

Considerando que o Mestrado Nacional Profissional em Ensino de Física objetiva a melhoria da "[...] qualificação profissional de professores de Física em exercício na Educação Básica visando tanto ao desempenho do professor no exercício de sua profissão como ao desenvolvimento de técnicas e produtos para a aprendizagem de Física" (SOCIEDADE BRASILEIRA DE FÍSICA, 2015, p. 1), entendemos que o processo de seleção para o ingresso no MNPEF deve ser pautado em critérios classificatórios, uma vez que os candidatos para ingresso estão habilitados (ou em fase de conclusão do curso de formação inicial) e exercendo as atribuições de um professor de Física. 
Para melhor representarmos nossa percepção, Nascimento (2013) nos mostrou que, no primeiro processo seletivo do Mestrado Nacional Profissional em Ensino de Física, 711 candidatos realizaram a prova escrita nacional, dos quais 390 foram classificados para a segunda fase. Ao final deste processo seletivo, registrou-se $14,5 \%$ de vagas ociosas (vagas ofertadas que não foram preenchidas). Ou seja, algumas das vagas abertas no primeiro edital do Mestrado Nacional Profissional em Ensino de Física não foram preenchidas em razão de eliminações precoces de candidatos que não tiveram o desempenho esperado pela Comissão de Pós-Graduação Nacional na prova escrita nacional, mas que possivelmente estavam atuando como professores de Física na Educação Básica. Nesse contexto, o processo seletivo se apresenta como uma questão a ser melhor discutida no Mestrado Nacional Profissional em Ensino de Física, em especial, sobre a prova escrita nacional ser pautada exclusivamente em questões de conteúdos de Física, ser centralizada (elaborada pela Comissão de PósGraduação nacional sem qualquer participação dos Polos Regionais) e possuir caráter eliminatório.

Pensando nos professores titulados mestres em Ensino de Física pelo Mestrado Nacional Profissional em Ensino de Física, temos que, passados os cinco primeiros anos do programa, somente as turmas formadas nos três primeiros editais podem ter concluintes. Assim, denominaremos essas três turmas pelo ano e semestre de início das atividades acadêmicas: 2013/2, 2014/2 e 2015/1.

Como o MNPEF (2018) não disponibiliza em sua página da internet um sistema on-line para consulta de informações sobre os discentes e docentes do programa, pesquisamos nos sites de cada Polo Regional as informações que precisávamos referentes ao número total de concluintes nestas três turmas. Quando não estavam disponíveis nos sites dos próprios Polos Regionais, entramos em contato, via e-mail, com as coordenações de cada Polo Regional solicitando tais informações. Ainda assim, houve casos de Polo Regional que não nos deram retorno, de modo que a partir do nome dos candidatos aprovados nos processos seletivos foi possível buscar as informações que nos faltavam no Currículo Lattes ${ }^{4}$ e no Catálogo de Teses e Dissertações da Capes ${ }^{5}$ até setembro de 2018.

Na tabela 1, apresentamos o número de vagas ofertadas e de concluintes de acordo com cada Polo Regional do Mestrado Nacional Profissional em Ensino de Física que participou de algum dos três primeiros processos seletivos. Vale lembrarmos que neste estudo, não estamos preocupados em desenvolver uma análise minuciosa sobre o desempenho acadêmico dos discentes/professores do Mestrado Nacional Profissional em Ensino de Física. Mesmo porque, nos falta informações como o número de matriculados em cada turma. Em essência, nosso objetivo aqui é apenas destacar o quantitativo de professores titulados Mestre em Ensino de Física pelo Mestrado Nacional Profissional em Ensino de Física para ressaltar o potencial de formação em larga escala deste programa.

${ }^{4}$ Disponível em: http://lattes.cnpq.br/. Disponível em: 20 jun. 2020.

${ }^{5}$ Disponível em: http://catalogodeteses.capes.gov.br/. Disponível em: 20 jun. 2020. 

Tabela 1 - quantitativo da oferta de vagas e de concluintes para cada Polo Regional dos três primeiros
editais de processo seletivo do Mestrado Nacional Profissional em Ensino de Física

\begin{tabular}{|c|c|c|c|c|c|c|}
\hline \multirow{3}{*}{ Sigla* } & \multicolumn{3}{|c|}{ Número de vagas ofertadas } & \multirow{2}{*}{\multicolumn{3}{|c|}{$\begin{array}{l}\text { Concluintes do curso } \\
\text { Editais MNPEF-SBF }\end{array}$}} \\
\hline & \multicolumn{3}{|c|}{ Editais MNPEF-SBF } & & & \\
\hline & $1 / 2013$ & $1 / 2014$ & $1 / 2015$ & $1 / 2013$ & $1 / 2014$ & $1 / 2015$ \\
\hline PR 01 & 15 & 20 & 0 & 22 & 11 & 0 \\
\hline PR 02 & 15 & 15 & 0 & 13 & 6 & 0 \\
\hline PR 03 & 10 & 10 & 0 & 8 & 4 & 0 \\
\hline PR 04 & 20 & 10 & 0 & 12 & 3 & 0 \\
\hline PR 05 & 15 & 10 & 0 & 13 & 8 & 0 \\
\hline PR 06 & 20 & 10 & 0 & 7 & 4 & 0 \\
\hline PR 07 & 15 & 0 & 10 & 8 & 0 & 0 \\
\hline PR 08 & 15 & 0 & 10 & 9 & 0 & 8 \\
\hline PR 09 & 15 & 0 & 10 & 15 & 0 & 9 \\
\hline PR 10 & 20 & 0 & 10 & 18 & 0 & 7 \\
\hline PR 11 & 20 & 0 & 10 & 8 & 0 & 7 \\
\hline PR 12 & 15 & 10 & 0 & 13 & 9 & 0 \\
\hline PR 13 & 15 & 10 & 0 & 11 & 8 & 0 \\
\hline PR 14 & 10 & 5 & 0 & 5 & 1 & 0 \\
\hline PR 15 & 30 & 10 & 0 & 19 & 9 & 0 \\
\hline PR 16 & 20 & 13 & 0 & 15 & 9 & 0 \\
\hline PR 17 & 30 & 20 & 0 & 11 & 14 & 0 \\
\hline PR 18 & 10 & 0 & 0 & 8 & 0 & 0 \\
\hline PR 19 & 15 & 15 & 0 & 4 & 5 & 0 \\
\hline PR 20 & 10 & 10 & 0 & 6 & 9 & 0 \\
\hline PR 21 & 15 & 0 & 0 & 9 & 0 & 0 \\
\hline PR 22 & 0 & 10 & 0 & 0 & 9 & 0 \\
\hline PR 23 & 0 & 15 & 0 & 0 & 14 & 0 \\
\hline PR 24 & 0 & 15 & 0 & 0 & 13 & 0 \\
\hline PR 25 & 0 & 20 & 0 & 0 & 13 & 0 \\
\hline PR 26 & 0 & 10 & 0 & 0 & 7 & 0 \\
\hline PR 27 & 0 & 10 & 0 & 0 & 8 & 0 \\
\hline PR 28 & 0 & 10 & 0 & 0 & 6 & 0 \\
\hline PR 29 & 0 & 12 & 0 & 0 & 8 & 0 \\
\hline PR 30 & 0 & 10 & 0 & 0 & 8 & 0 \\
\hline PR 31 & 0 & 10 & 0 & 0 & 9 & 0 \\
\hline PR 32 & 0 & 10 & 0 & 0 & 9 & 0 \\
\hline PR 33 & 0 & 15 & 0 & 0 & 12 & 0 \\
\hline PR 34 & 0 & 10 & 0 & 0 & 6 & 0 \\
\hline PR 35 & 0 & 15 & 0 & 0 & 15 & 0 \\
\hline PR 36 & 0 & 10 & 0 & 0 & 8 & 0 \\
\hline PR 37 & 0 & 12 & 0 & 0 & 11 & 0 \\
\hline PR 38 & 0 & 15 & 0 & 0 & 5 & 0 \\
\hline PR 39 & 0 & 20 & 0 & 0 & 15 & 0 \\
\hline PR 40 & 0 & 15 & 0 & 0 & 7 & 0 \\
\hline PR 41 & 0 & 10 & 0 & 0 & 10 & 0 \\
\hline PR 42 & 0 & 15 & 0 & 0 & 9 & 0 \\
\hline PR 43 & 0 & 15 & 0 & 0 & 5 & 0 \\
\hline PR 44 & 0 & 20 & 0 & 0 & 8 & 0 \\
\hline PR 45 & 0 & 11 & 0 & 0 & 5 & 0 \\
\hline PR 46 & 0 & 0 & 15 & 0 & 0 & 8 \\
\hline Total & 365 & 483 & 65 & 234 & 320 & 39 \\
\hline
\end{tabular}

*PR $=$ Polo Regional.

Fonte: elaborado pelos autores. 
Inicialmente, em uma análise absoluta dos números, das 913 vagas ofertas para as três primeiras turmas do Mestrado Nacional Profissional em Ensino de Física, temos que 593 professores foram titulados Mestre em Ensino de Física. Em termos de percentual, 65\% do total de vagas ofertadas culminaram com a conclusão do curso por parte dos discentes/ professores.

Nesta análise, importa-nos esclarecer que os 35\% que não concluíram o curso não são unicamente casos de evasão, isto é, discente/professores que se matricularam em algum Polo Regional do Mestrado Nacional Profissional em Ensino de Física e não concluíram o curso. Neste percentual, temos também as vagas ociosas, que não foram preenchidas ou por número inferior de candidatos inscritos ou por número inferior de candidatos aprovados em relação ao número de vagas ofertadas nos processos seletivos. De qualquer forma, pensando em investigações futuras, parece-nos indispensável uma avaliação sobre os discentes/ professores nessa direção, principalmente para refletirmos sobre possíveis contornos para o não preenchimento das vagas ofertadas nos Polos Regionais.

Para além, merece nossa atenção os egressos do Mestrado Nacional Profissional em Ensino de Física, no sentido de investigarmos os impactos desse programa na formação pessoal e no trabalho docente destes egressos na Educação Básica. Ou seja, quais as possíveis mudanças percebidas pelos próprios egressos no seu trabalho docente (em sua dinâmica de sala de aula)? Uma vez não sendo tão valorizado a titulação acadêmica do professor na Educação Básica, como por exemplo nos Institutos Federais de Educação, Ciência eTecnologia e nas Universidades, estes egressos permanecem na Educação Básica?

Por fim, outra questão que se apresenta diz respeito à produção acadêmica do Mestrado Nacional Profissional em Ensino de Física, por vezes pensados com grande potencial de aplicação nas salas de aula. Nesse sentido, devemos seguir com pesquisas que tomaram como objeto esse tipo de produção acadêmica em outros contextos (NASCIMENTO; OSTERMANN; CAVALCANTI, 2017) e no próprio contexto do Mestrado Nacional Profissional em Ensino de Física (ANTUNES JR; OSTERMANN; CAVALCANTI, 2019). Tais pesquisas evidenciam o descompasso entre o que se produz na acadêmica, produtos educacionais com um viés meramente instrumentalista, e as necessidades escolares.

\section{Considerações Finais}

Neste artigo, analisamos os cursos de Mestrado Profissional em Ensino como cursos de formação continuada de professores no contexto da Nova Gestão Pública e de suas práticas presentes na gestão educacional. Neste cenário, apontamos algumas das mudanças ocorridas na formação e no trabalho docente, destacando o modelo standard de formação continuada proposto pelos Mestrado Profissional em Ensino, em especial na subárea de Ensino de Ciências e Matemática, e a adoção de uma lógica empresarial, de desempenho e eficiência, para balizar o trabalho docente por meio da produção e aplicação de produtos educacionais.

Passados alguns anos, os cursos de Mestrado Profissional em Ensino se consolidaram no Sistema Nacional de Pós-Graduação e, de certa forma, oportunizaram o surgimento dos programas em rede nacional. O que por sua vez possibilitou a ampliação da oferta de vagas para os cursos de Mestrado Profissional em Ensino, reafirmando, assim, o potencial de oferta em larga escala destes cursos e ao mesmo tempo, o modelo standard de formação, sobretudo com a prerrogativa de um currículo nacional a ser adotado nos mais diversos Polos Regionais que formam um determinado programa em rede nacional. 
No caso do Mestrado Nacional Profissional em Ensino de Física, após cinco anos de sua criação, além de apresentarmos as principais características, tais como Regimento Geral (SOCIEDADE BRASILEIRA DE FÍSICA, 2015) e Matriz Curricular Nacional (SOCIEDADE BRASILEIRA DE FÍSICA, 2014), fizemos uma análise com base no número de vagas ofertadas, via editais de seleção para ingresso, e no número de discentes/professores concluintes do curso. Com isso, identificamos que nas três primeiras turmas formadas no Mestrado Nacional Profissional em Ensino de Física, 913 vagas foram ofertadas, das quais 593 culminaram com a titulação de professores. De fato, com 60 Polos Regionais integrados ao programa, dentro da lógica empresarial que busca a gestão de desempenho, o MNPEF tem correspondido com o seu objetivo de promover a formação continuada de professores de Física em larga escala. Entretanto, nos parece indispensável uma reflexão sobre os processos de criação e administração deste programa, que, além de alinhados às práticas da Nova Gestão Pública, estão em domínio de um único grupo acadêmico.

Enquanto objeto de investigação, ainda em 2014, iniciamos um programa de pesquisa sobre o Mestrado Nacional Profissional em Ensino de Física (ANTUNES JR., 2018; REBEQUE, 2017), de modo a investigarmos inúmeros aspectos envolvidos no interior desse fenômeno social e complexo. Em especial, para o contexto de construção e implementação do programa em nível nacional e local (dos Polos Regionais), para a produção acadêmica dos discentes/ professores, dissertações e produtos educacionais. Todo esse corpo de conhecimento que produzimos teve como base as pesquisas de Schäffer (2013), Souza (2015) e Nascimento (2016), entre outras, sobre o programa de mestrado profissional pioneiro na área de Ensino de Física, o já mencionado MPEF da UFRGS, tendo em vista que este curso serviu de base para o projeto do Mestrado Nacional Profissional em Ensino de Física.

Entendemos que o Mestrado Nacional Profissional em Ensino de Física deveria ser avaliado institucionalmente pelas instâncias que regulam a pós-graduação Brasil, com critérios claros e, possivelmente, estabelecidos com base nas dinâmicas de avaliação para os cursos de Mestrado Profissional em Ensino do Sistema Nacional de Pós-Graduação. Passados cinco anos de existência do MNPEF, não temos informações de como (quais critérios e dinâmicas) ou por quem (pela própria Sociedade Brasileira de Física, pela Coordenação de Aperfeiçoamento de Pessoal de Nível Superior, ou ainda, por uma comissão externa) será feita a avaliação do Mestrado Nacional Profissional em Ensino de Física. Ademais, caso a avaliação seja feita por alguma área do Sistema Nacional de Pós-Graduação da CAPES, qual área terá essa responsabilidade: Física ou Ensino?

Para encerrar, pensando na continuidade do Mestrado Nacional Profissional em Ensino de Física, entendemos que se faz necessário discussões em dois níveis: a primeira interna, de pensarmos na abertura da gestão administrativa do programa por parte da Sociedade Brasileira de Física, e a segunda, de nível amplo, sobre as conexões do MNPEF, e os demais programas em rede nacional, com as políticas públicas de valorização da carreira dos professores. Por um lado, no nível interno, há de se pensar uma abertura do regimento e do currículo nacional para que os Polos Regionais do Mestrado Nacional Profissional em Ensino de Física, com as experiências adquiridas nestes primeiros anos, possam ter autonomia e desenvolverem dinâmicas formativas não padronizadas e que fogem ao academicismo. Além disso, devemos pensar em uma administração plural, com os órgãos administrativos do programa sendo eleito pelos pares (sócios da Sociedade Brasileira de Física e comunidade acadêmica envolvida nos Polos Regionais) e não nomeada exclusivamente pelo Conselho da Sociedade Brasileira de Física. Por outro lado, pensando em termos de macropolítica, os 
programas de mestrado profissional em rede nacional precisam de melhor integração com as demais políticas públicas relativas à carreira da profissão docente, tanto as precedentes, como as políticas de formação inicial de professores, como as subsequentes, principalmente de progressão funcional na carreira e de reajuste salarial conforme titulação, dentre outros aspectos de natureza estrutural.

\section{Referências}

ANDERSON, G. Privatizando subjetividades: como a nova gestão pública está criando o "novo" profissional da educação. Revista Brasileira de Política e Administração da Educação, Goiânia, v. 33, n. 3, p. 593-626, 2017.

ANTUNES JR., E. L. Q. Formação de professores em larga escala: um estudo sobre o mestrado nacional profissional em ensino de física. 2018. Dissertação (Mestrado em Ensino de Física) - Universidade Federal do Rio Grande do Sul, Porto Alegre, 2018.

ANTUNES JR., E. L.; OSTERMANN, F.; CAVALCANTI, C. J. H. A subvalorização da formação continuada de professores: dos orientadores à articulação do referencial teórico no contexto do MNPEF. Alexandria, Florianópolis, v. 12, n. 2, p. 267-291, 2019.

BRASIL. Portaria n 131, de 28 de Junho de 2017. Dispõe sobre o mestrado e doutorado profissionais. Diário Oficial da União: seção 1, Brasília, ed. 124, p. 17, 30 jun. 2017.

BRASIL. Portaria $n^{\circ}$ 60, de 20 de Março de 2019. Dispõe sobre o mestrado e doutorado profissionais, no âmbito da Coordenação de Aperfeiçoamento de Pessoal de Nível Superior - CAPES. Diário Oficial da União: seção 1, Brasília, ed. 56, p. 26, 22 mar. 2019.

CAPES. Relatório de avaliação: ensino. Relatório de avaliação 2013-2016. Brasília: Capes, 2017. Disponível em: https://tinyurl.com/y6vydlbd. Acesso em: 11 maio de 2018.

CASAGRANDE, I. M. K.; PEREIRA, S. M.; SAGRILLO, D. R. O Banco Mundial e as políticas de formação docente no Brasil. Educação Temática Digital, Campinas, v. 16, n. 3, p. 494-512, 2014.

CÓSSIO, M. F. A nova gestão pública: alguns impactos nas políticas educacionais e na formação professores. Educação, Porto Alegre, v. 41, n. 1, p. 63-73, 2018.

DIAS, R. E.; LOPES, A. C. Competências na formação de professores no Brasil: o que (não) há de novo. Educação \& Sociedade, Campinas, v. 24, n. 85, p. 1155-1177, 2003.

EVANGELISTA, O.; SHIROMA, E. O. Professor: protagonista e obstáculo da reforma. Educação e Pesquisa, São Paulo, v. 33, n. 3, p. 531-541, 2007.

FREITAS, H. C. L. A (nova) política de formação de professores: a prioridade postergada. Educação \& Sociedade, Campinas, v. 28, n. 100, p.1203-1230, 2007.

IMBERNÓN, F. Formação continuada de professores. Porto Alegre: Artmed, 2010.

KUENZER, A. Z. A formação de professores para o ensino médio: problemas e desafios. Educação \& Sociedade, Campinas, v. 32, n. 116, p. 667-688, 2011.

LIMA, K. R. S. O Banco Mundial e a educação superior brasileira na primeira década do novo século. Katálysis, Florianópolis, v. 14, n. 1, p. 86-94, 2011.

MAUÉS, O. C. Reformas internacionais da educação e formação de professores. Cadernos de Pesquisa, São Paulo, n. 118, p. 89-117, 2003. 
MNPEF: Mestrado profissional em ensino de física. Disponível em: http://www1.fisica.org.br/ mnpef/?q=apresentação. Acesso em 13 set. 2018.

MOREIRA, M. A. O mestrado (profissional) em ensino. Revista Brasileira de Pós-Graduação, Brasília, v. 1, n. 1, p. 131-142, 2004.

MOREIRA, M. A. Orientações sobre o currículo do MNPEF. [S.I.: s.n.], 2015. Disponível em: https:// tinyurl.com/y9epd2kd. Acesso em: 2 out. 2018.

MOREIRA, M. A.; NARDI, R. O mestrado profissional na área de ensino de ciências e matemática: alguns esclarecimentos. Revista Brasileira de Ensino de Ciência e Tecnologia, Curitiba, v. 2, n. 3, p. 1-9, 2009.

MOREIRA, M. A.; STUDART, N. Comunicado 09/2015. São Paulo: Sociedade Brasileira de Física, 2015. Disponível em: https://tinyurl.com/y6vs5yur. Acesso em: 2 out. 2018.

MOREIRA, M. A.; STUDART, N.; VIANNA, D. M. O mestrado nacional profissional em ensino de física (MNPEF): uma experiência em larga escala no Brasil. Latin-American Journal of Physics Education, México, v. 10, n. 4, p. 4327-1/4327-6, 2016.

NASCIMENTO, M. M. Análise de produtos educacionais desenvolvidos no âmbito de um mestrado profissional em ensino de física. 2016. Dissertação (Mestrado em Ensino de Física) - Universidade Federal do Rio Grande do Sul, Porto Alegre, 2016.

NASCIMENTO, M. M.; OSTERMANN, F.; CAVALCANTI, C. J. H. Análises multidimensional e Bakhtiniana do discurso de trabalhos de conclusão desenvolvidos no âmbito de um mestrado profissional em ensino de física. Ciência \& Educação, Bauru, v. 23, n. 1, p. 181-196, 2017. DOI: http://doi.org/d2h2.

NASCIMENTO, S. S. O mestrado nacional profissional de ensino em física: a experiência da Sociedade Brasileira de Física. Polyphonía, Goiânia, v. 24, n. 2, p. 265-268, 2013.

OLIVEIRA, D. A. Nova gestão pública e governos democrático-populares: contradições entre a busca da eficiência e a ampliação do direito à educação. Educação \& Sociedade, Campinas, v. 36, n. 132, p. 625-646, 2015.

OSTERMANN, F.; REZENDE, F. Projetos de desenvolvimento e de pesquisa na área de ECM: uma reflexão sobre os mestrados profissionais. Caderno Brasileiro de Ensino de Física, Florianópolis, v. 26, n. 1, p. 66-80, 2009.

REBEQUE, P. V. Políticas públicas de formação continuada de professores: investigações sobre o mestrado nacional profissional em ensino de física. 2017. Tese (Doutorado em Ensino de Física) Universidade Federal do Rio Grande do Sul, Porto Alegre, 2017.

REBEQUE, P. V.; OSTERMANN, F.; VISEU, S. O mestrado nacional profissional em ensino de física: investigando os modos de regulação em um polo regional do programa. Revista Brasileira de Pesquisa em Educação em Ciências, São Paulo, v. 18, n. 2, p. 399-428, 2018.

REBEQUE, P. V.; OSTERMANN, F.; VISEU, S. Os mestrados profissionais em ensino de ciências e matemática no Brasil: um tema pouco explorado na literatura. Revista Brasileira de Ensino de Ciência e Tecnologia, Curitiba, v. 10, n. 2, p. 1-21, 2017.

ROBERTSON, S. L. As implicações em justiça social da privatização nos modelos de governança da educação: um relato relacional. Educação \& Sociedade, Campinas, v. 34, n.345, p. 679-703, 2013.

SANTOS, L. L. C. P.; DINIZ-PEREIRA, J. E. Tentativas de padronização do currículo e da formação de professores no Brasil. Cadernos CEDES, Campinas, v. 36, n. 100, p. 281-300, 2016. 
SAUERWEIN, I. P. S.; DELIZOICOV, D. Formação continuada de professores de física no ensino médio: concepções de formadores. Caderno Brasileiro de Ensino de Física, Florianópolis, v. 25, n. 3, p. 439$477,2008$.

SOCIEDADE BRASILEIRA DE FÍSICA. Grade curricular 2014: MNPEF-SBF. [São Paulo: SBF], 2014. Disponível em: https://tinyurl.com/yafn9kor. Acesso em: 2 out. 2018.

SOCIEDADE BRASILEIRA DE FÍSICA. Regimento geral do mestrado nacional profissional em ensino de física. [São Paulo: SBF], 2015. Disponível em: https://tinyurl.com/ybgxl475. Acesso em: 2 out. 2018.

SCHÄFER, E. D. A. Impacto do mestrado profissional em ensino de física da UFRGS na prática docente: um estudo de caso. 2013. Tese (Doutorado em Ensino de Física) - Universidade Federal do Rio Grande do Sul, Porto Alegre, 2013.

SOUZA, J. Apropriação discursiva de modelos de formação em trabalhos de conclusão de um mestrado profissional em ensino de física. 2015. Dissertação (Mestrado em Ensino de Física) - Universidade Federal do Rio Grande do Sul, Porto Alegre, 2015.

VILLANI, A. Mestrado profissional em ensino de ciências e matemática: uma interpretação. Revista Ibero-Americana de Estudos em Educação, Araraquara, v. 11, n. esp. 1, p. 418-433, 2016.

VILLANI, A.; BAROLLI, E.; MAIA, J. O.; M ASSI, L.; SANTOS, V. F. D.; NASCIMENTO, W. E. Mestrados profissionais em ensino de ciências: estrutura, especificidade, efetividade e desenvolvimento profissional docente. Investigações em Ensino de Ciências, Porto Alegre, v. 22, n. 1, p. 127-161, 2017.

Anexo A - Detalhamento dos 60 Polos Regionais (PR) que integram o Mestrado Nacional Profissional em Ensino de Física (MNPEF)

\begin{tabular}{|c|c|c|c|}
\hline \multirow{22}{*}{ 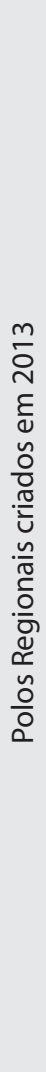 } & Sigla & Instituição de Ensino Superior (IES) & Município/Estado \\
\hline & PR 01 & Universidade de Brasília (UnB) & Distrito Federal/DF \\
\hline & PR 02 & Universidade Federal do Goiás (UFG) & Catalão/GO \\
\hline & PR 03 & Universidade Federal de Mato Grosso (UFMT) & Barra do Garça/MT \\
\hline & PR 04 & Universidade Federal do Amazonas (UFAM) e Instituto Federal do Amazonas (IFAM) & Manaus/AM \\
\hline & PR 05 & Universidade Federal de Rondônia (UNIR) & Jí-Paraná/RO \\
\hline & PR 06 & Universidade Estadual de Feira de Santana (UEFS) & Feira de Santana/BA \\
\hline & PR 07 & Universidade Federal Rural de Pernambuco (UFRPE) & Garanhuns/PE \\
\hline & PR 08 & Universidade Federal do Vale do São Francisco (UNIVASF) & Juazeiro/BA \\
\hline & PR 09 & Universidade Federal Rural do Semi-Árido (UFERSA) & Mossoró/RN \\
\hline & PR 10 & Instituto Federal do Rio Grande do Norte (IFRN) & Natal/RN \\
\hline & PR 11 & Universidade Federal de Sergipe (UFS) & São Cristóvão/SE \\
\hline & PR 12 & Universidade Federal do Espírito Santo (UFES) & Vitória/ES \\
\hline & PR 13 & Universidade Federal de Lavras (UFLA) & Lavras/MG \\
\hline & PR 14 & Universidade Federal de Viçosa (UFV) & Viçosa/MG \\
\hline & PR 15 & Universidade Federal Fluminense (UFF) & Volta Redonda/RJ \\
\hline & PR 16 & Universidade Estadual Paulista (UNESP) & Presidente Prudente/SP \\
\hline & PR 17 & Universidade Federal do $A B C$ (UFABC) & Santo André/SP \\
\hline & PR 18 & Universidade Federal de São Carlos (UFSCar) & São Carlos/SP \\
\hline & PR 19 & Universidade Estadual de Londrina (UEL) & Londrina/PR \\
\hline & PR 20 & Universidade Estadual de Maringá (UEM) & Maringá/PR \\
\hline & PR 21 & Universidade Federal de Rio Grande (FURG) & Rio Grande/RS \\
\hline
\end{tabular}




\begin{tabular}{|c|c|c|c|}
\hline \multirow{24}{*}{ 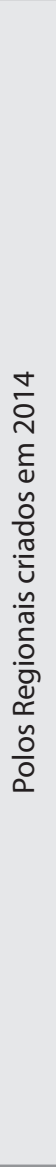 } & PR 22 & Universidade Federal do Rio de Janeiro (UFRJ) & Rio de Janeiro/RJ \\
\hline & PR 23 & Universidade Estadual do Ceará (UFCE) & Quixadá/CE \\
\hline & PR 24 & Universidade Federal de Juiz de Fora (UFJF) & Juiz de Fora/MG \\
\hline & PR 25 & Universidade Federal de Mato Grosso (UFMT) & Cuiabá/MT \\
\hline & PR 26 & Universidade Federal do Piauí (UFPI) & Teresina/PI \\
\hline & PR 27 & Universidade Federal do Rio de Janeiro (UFRJ) & Macaé/RJ \\
\hline & PR 28 & Universidade Federal de Alfenas (UNIFAL) & Alfenas/MG \\
\hline & PR 29 & Universidade Federal do Sul e Sudeste do Pará (UNIFESSPA) & Marabá/PA \\
\hline & PR 30 & Universidade Federal do Estado do Rio de Janeiro (UNIRIO) & Rio de Janeiro/RJ \\
\hline & PR 31 & Universidade Regional do Cariri (URCA) & Juazeiro do Norte/CE \\
\hline & PR 32 & Universidade Tecnológica Federal do Paraná (UTFPR) & Campo Mourão/PR \\
\hline & PR 33 & Instituto Federal do Espirito Santo (IFES) & Cariacica/ES \\
\hline & PR 34 & Instituto Federal Fluminense (IFF) & Campos dos Goytacazes/RJ \\
\hline & PR 35 & Universidade Estadual de Ponta Grossa (UEPG) & Ponta Grossa/PR \\
\hline & PR 36 & Universidade Federal De Alagoas (UFAL) & Maceió/AL \\
\hline & PR 37 & Universidade Federal do Pará (UFPA) & Belém/PA \\
\hline & PR 38 & Universidade Federal de Roraima (UFRR) & Boa Vista/RR \\
\hline & PR 39 & Universidade Federal de Santa Catarina (UFSC) & Florianópolis/SC \\
\hline & PR 40 & Universidade Federal de Rondônia (UNIR) & Porto Velho/RO \\
\hline & PR 41 & Universidade Federal de Santa Catarina (UFSC) & Araranguá/SC \\
\hline & PR 42 & Universidade Federal de São Carlos (UFSCar) & Sorocaba/SP \\
\hline & PR 43 & Universidade Federal do Ceará (UFCE) & Fortaleza/CE \\
\hline & PR 44 & Universidade Estadual de Santa Cruz (UESC) & Ilhéus/BA \\
\hline & PR 45 & Universidade Federal da Grande Dourados (UFGD) & Dourados/MS \\
\hline \multirow{15}{*}{ 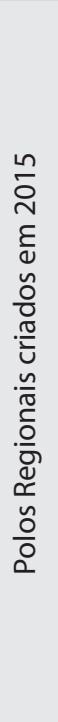 } & PR 46 & Universidade Federal de Pernambuco (UFPE) & Caruaru/PE \\
\hline & PR 47 & Universidade Federal do Maranhão (UFMA) & São Luís/MA \\
\hline & PR 48 & Universidade Estadual da Paraíba (UEPB) & Campina Grande/PB \\
\hline & PR 49 & Universidade Federal do Oeste do Pará (UFOPA) & Santarém/PA \\
\hline & PR 50 & Universidade Federal do Rio Grande do Sul (UFRGS) & Tramandaí/RS \\
\hline & PR 51 & Universidade Federal do Rio Grande do Norte (UFRN) & Natal/RN \\
\hline & PR 52 & Universidade Federal de Santa Catarina (UFSC) & Blumenau/SC \\
\hline & PR 55 & Universidade Tecnológica Federal do Paraná (UTFPR) & Medianeira/PR \\
\hline & PR 56 & Instituto Federal do Ceará (IFCE) & Sobral/CE \\
\hline & PR 58 & Universidade Federal Rural de Pernambuco (UFRPE) & Recife/PE \\
\hline & PR 59 & Universidade Federal Do Acre (UFAC) & Rio Branco/AC \\
\hline & PR 60 & Universidade do Estado da Bahia (UNEB) & Salvador/BA \\
\hline & PR 61 & Universidade Federal do Tocantins (UFT) & Araguaína/TO \\
\hline & PR 62 & Universidade Estadual do Sudoeste da Bahia (UESB) & Vitória da Conquista/BA \\
\hline & PR 63 & Instituto Federal do Maranhão (IFMA) & São Luís/MA \\
\hline
\end{tabular}

Fonte: elaborado pelos autores. 